\title{
Gestión de Riesgos de Desastres en Albergues en el distrito del Rimac ${ }^{1}$
}

\author{
Disaster Risk Management in Shelter in RIMAC district
}

Eric Espinoza Campos ${ }^{2}$

\section{RESUMEN}

En agosto del 2016, el Municipio Distrital del Rímac (MDR) aprueba un Plan de Rehabilitación ante Riesgo de Desastre del distrito del Rímac.- En noviembre del 2016, se produce un incendio en el sector de Cantagallo en el distrito del Rímac resultando 300 familias afectadas/damnificadas de la comunidad Shipibo-Coniba quienes reciben la ayuda humanitaria de parte de la Municipalidad del Rímac y de la Municipalidad Metropolitana de Lima (MML).- La etapa post-desastre en este caso ha demostrado la dificultad de ayuda oportuna a un sector de ciudadanos del distrito del Rímac. Una variable es la ubicación de espacios públicos para reubicar personas, porque se destinó un espacio de albergue en el denominado campo de MARTINETI en el distrito del Cercado de Lima, es decir encontrar una solución en otro distrito. Otra variable es el bajo nivel de coordinación de los funcionarios entre entidades estatales. Un factor de ayuda fue que la población afectada /damnificada estaba concentrada en un sector, y pertenece a una misma comunidad cultural. Este factor facilita la comunicación y las coordinaciones con los funcionarios públicos. La

\footnotetext{
1 Origen Los albergues en la etapa post-desastre presentan dificultades que se evidencian por su larga duración en Pisco Perú después del sismo del 2007, y por su ubicación de afectados/damnificados de un distrito a otro en un evento adverso de menor magnitud como un incendio en la zona de Cantagallo en 2016 en la ciudad de Lima. Entonces se requiere analizar esta situación con sus variables a identificar para mejorar la operatividad de este componente de albergue dentro de una Gestión de Riesgo de Desastre.

Tipo de artículo de reflexión. Metodología cualitativa

2 Ingeniero Industrial, Universidad Nacional de Ingeniería, Lima, Perú. Egresado de Maestría de Gestión Económica de Riesgo de Desastres y Desarrollo Sostenible Universidad Nacional Mayor de San Marcos en Lima, Perú. Correo electrónico: Eric.Espinoza.C@ciplima.org.pe
}

(C) Los autores. Este artículo es publicado por Pensamiento Crítico de la Facultad de Ciencias Económicas, Universidad Nacional Mayor de San Marcos. Este es un artículo de acceso abierto, distribuido bajo los términos de la licencia Creative Commons Atribucion - No Comercia_Compartir Igual 4.0 Internacional. (http://creativecommons.org/licenses/by-nc-sa/4.0/) que permite el uso no comercial, distribución y reproducción en cualquier medio, siempre que la obra original sea debidamente citada. 
actual situación de zonas de albergue/refugio en la MDR necesita ser corregida por personal calificado.

PALABRAS CLAVES: Albergue; desastre; espacios; ayuda; calidad de vida; Proyecto Esfera.

JEL: 029, Q54, Z19.

\section{ABSTRAC}

In 2016, the MDR approve Rehabilitation Plan for Disaster Risk in Rimac district. -In 2016, there is a fire in the community of CANTAGALLO in the district of Rimac. 300 families of the SHIPIBO-CONIBA community are affected, who receive the support from the Municipality of Rimac and the Metropolitan Municipality of Lima. -The Disaster Risk Management employees in Rimac district spend five years to elaborate a rehabilitation plan. This harmful event happened in a three-month short-time. -The post-disaster stage in this case has shown the difficulty of helping a number of citizen in the district of RIMAC. The issue is the location of public spaces to relocate people, because it has had to occupy a space in the so-called MARTINETI field in the district of Cercado de Lima that is to find a solution in another district. This is only part of the problem. The coordination of the officials of a district municipality with the metropolitan municipality of Lima is a serious factor. A help factor is that those affected are from a cultural community, this factor has facilitated understanding between the authorities and the affected population. -The current situation for shelter areas requires a real management for qualified professionals.

KEY WORDS: Shelter; disaster; spaces; supplies; life quality; Sphere Project. JEL: 029, Q54, Z19.

\section{La población en el Rímac}

En el 2018, la ciudad de Lima, situada en la provincia y departamento de Lima, tiene 483 años de fundación y en este tiempo ha experimentado una expansión urbana de tipo horizontal y vertical. Este crecimiento no implica un desarrollo económico homogéneo en la ciudad.

En un recorrido físico por las principales avenidas de la ciudad de Lima puede verificarse edificaciones multifamiliares, centros comerciales como prueba del crecimiento vertical de la ciudad. Actualmente, la ciudad de Lima tiene 43 distritos.

En el 2018, el censo del Instituto Nacional de Estadísticas e Informática (INEI) XII de Población, VII de Vivienda y III de Comunidades Indíge- 
nas da como primeras cifras una población nacional de 31 millones 237 mil 385 y el departamento más poblado es Lima con más de 9 millones de habitantes, es decir el 30\%. Por temas de control de calidad de datos en el INEI aún no hay resultados finales por eso este artículo usa la data censual del 2007. (25 de junio del 2018.Nota de Prensa $N^{\circ} 108$ Población del Perú totalizó 31 millones 237 mil 385 personas al 2017. INEI.)

El distrito del Rímac no puede expandirse horizontalmente porque está restringido o encerrado en la zona centro de la ciudad así que su expansión urbana se limita en forma vertical. Además, el Cercado de Lima y el distrito del Rímac tienen la categoría de ser patrimonio histórico cultural y esta condición cultural es un factor limitante para cambios y/o modificaciones urbanísticas.

En 1998, la Organización de las Naciones Unidas para la Educación, la Ciencia y la Cultura (UNESCO) declara como Patrimonio de la Humanidad el Centro Histórico de Lima, responsabilidad compartida por la Municipalidad de Lima y la municipalidad del Rímac. (15 de abril del 2015. "Incluyen al Rímac en grupo de ciudades Patrimonio Mundial de UNESCO". Andina).

En la expansión urbana territorial de Lima hacia el Norte, Sur, y Este se denomina inicialmente Cono Norte, Sur y Este, luego se cambia la denominación a Lima Norte, Lima Sur y Lima Este. Por ejemplo, el distrito de Carabayllo está ubicado en la zona norte, San Juan de Lurigancho está en la zona este, y Villa El Salvador en la zona sur. (07 de octubre del 2018. Cono Sur y Cono Norte están en ruta de masificación de viviendas. Andina.).

Las tablas estadísticas $\mathrm{N}^{\circ} 1$ y $\mathrm{N}^{\circ} 2$ son comparativos de distritos por cada sector de Lima: (1) Del Centro (Lima-cercado y Rímac), (2) De Lima Norte (Carabayllo que es el distrito ubicado más hacia el Norte), (3) De Lima Este (San Juan de Lurigancho que es el distrito más hacia el Este) y (4) de Lima Sur (Villa El Salvador que es el distrito más hacia el Sur).

El distrito de San Juan de Lurigancho es el más poblado, y Carabayllo es el de mayor superficie territorial. El Rímac, es el distrito de menor superficie territorial y también el de menor población en la provincia de Lima. Véase las tablas $N^{\circ} 1$ y $N^{\circ} 2$. 
El distrito del Rímac posee el 2,3\% de la población y el 0,4 \% de la superficie y una densidad de áreas verdes de 1,07 $\mathrm{m} 2 /$ habitante en la provincia de Lima. Véase las tablas $N^{\circ} 1$ y $N^{\circ} 2$.

Tabla 1

Población y superficie: comparación referencial de distritos: 2007

\begin{tabular}{lcccccc} 
& \multicolumn{3}{c}{ Población (habitantes) } & \multicolumn{2}{c}{ Superficie Territorial (kms2) } \\
& $\mathbf{2 0 0 7}$ & $\mathbf{\%}$ & Ranking & $\mathbf{2 0 0 7}$ & $\mathbf{\%}$ & Ranking \\
\hline Provincia Lima & 7.605 .742 & 100,0 & & $2.664,67$ & 100,0 & \\
Lima Cercado & 299.493 & 4,0 & 9 & 21,98 & 0,8 & 23 \\
Rímac & 176.169 & 2,3 & 18 & 11,87 & 0,4 & 28 \\
Carabayllo & 213.386 & 2,8 & 13 & 346,88 & 13,0 & 1 \\
San Juan Lurigancho & 898.443 & 11,8 & 1 & 131,25 & 4,9 & 7 \\
Villa El Salvador & 381.790 & 5,0 & 5 & 35,46 & 1,3 & 19 \\
\hline
\end{tabular}

Fuente INEI Censo 2007 Perfil sociodemográfico p. 20,26 de 526.

Elaboración Propia

Tabla 2

Densidad poblacional por superficie, y densidad de áreas verdes por habitante 2007

\begin{tabular}{lccc} 
& área verde $\mathbf{m} 2$ & $\begin{array}{c}\text { Superficie } \\
\text { hab/km2 }\end{array}$ & $\begin{array}{c}\text { Área verde } \\
\text { M2/hab. }\end{array}$ \\
\hline Provincia Lima & 26.054 .085 & 2.854 & 3,43 \\
Lima Cercado & 657.001 & 13.626 & 2,19 \\
Rímac & 188.338 & 14.842 & 1,07 \\
Carabayllo & 573.911 & 615 & 2,69 \\
San Juan Lurigancho & 4.756 .000 & 6.845 & 5,29 \\
Villa El Salvador & 29,000 & 10.767 & 0,08 \\
\hline
\end{tabular}

Fuente INEI 2011 Provincia de Lima. Compendio Estadístico 2011-2012 p. 27 de 507.

Elaboración Propia

Un distrito tiene varios componentes urbanos como las áreas verdes, las escuelas, los centros de salud, las comisarías y proyectos de inversión, entre otros. Según experiencias históricas, las áreas verdes y escuelas se utilizan como zonas de albergue/refugio después de un evento adverso. 
A nivel internacional, la Organización Mundial de la Salud (OMS) establece un índice verde urbano (IVU) de $9 \mathrm{~m} 2$ de áreas verdes por persona. A nivel nacional, el Sistema Nacional de Información Ambiental del Ministerio del Ambiente (SINIA-MINAM) tiene calculado el Indicador de Superficie de área verde urbana por habitante para el departamento de Lima de 3,03 (año 2016). (MINAM. 2018. Indicador.)

Según el Instituto Metropolitano de Planificación (IMP) (2010. p 6-7 de 46) las áreas verdes que son las superficies privadas o públicas ocupadas por la vegetación y arboles tienen un valor/aporte múltiple para mejorar la calidad de vida de las personas dentro de la zona urbana. El aporte ambiental se logra con su vegetación y arboles regulando la intensidad de la radiación solar, la calidad del aire, y el atrapamiento de los gases tóxicos generados por el tráfico vehicular y la actividad industrial. En la salud mental y física aporta por su uso recreacional y en la interacción del habitante con una parte de la naturaleza. También aportan un valor económico porque vivir cercanamente a estas áreas aumenta el valor de los predios.

De los distritos representativos en Lima Norte, Sur, Este y Oeste e la Tabla 2 ninguno cumple con ese estándar del IVU de área verde por habitante, incluso el Rímac tiene el índice más bajo. Estos valores demuestran que las áreas verdes no cumplen con su valor/aporte múltiple. Entonces el uso forzado de estas zonas como albergue va a disminuir estos valores de IVU en corto plazo.

Un ejemplo de la extraña planificación de zonas verdes con las rutas de tránsito en el distrito del Rímac se aprecia en el parque Celso donde un parque es seccionado por una vía vehicular.

El Fondo de las Naciones Unidas para la Infancia (UNICEF) (2008, $p$ 1-68) recopiló y analizó una serie de casos en Bolivia y Nicaragua por el uso de las escuelas como zonas de albergue.

Asimismo, según UNICEF (2008, p 28 de 68 y 10 de 68) sostiene que en la etapa post-desastre cambian las prioridades de alimentación y educación en la atención a la niñez en las escuelas públicas. Por ejemplo, durante las inundaciones en Bolivia, era evidente el incremento del uso de las 
escuelas como refugios o albergues temporales. En Nicaragua, se autoriza el uso de escuelas como albergues temporales en caso de desastre.

El uso de escuelas públicas como albergues es polémico porque presenta una seria desventaja por el uso forzado de unas instalaciones que no tienen ese diseño.. Hay desarrollo en la protección de la niñez, pero se olvida que los docentes también tienen familia y es un factor que no es analizado.

Desde el 2016 entra en funcionamiento la obra de túneles Santa Rosa y San Martin de Porres que atraviesan el Cerro Santa Rosa y unen las rutas de tránsito vehicular de los distritos del Rímac y San Juan de Lurigancho, la zona centro y la zona Este. Estas vías de tráfico disminuyen el tiempo de viaje porque representa la distancia más corta entre ambos distritos y beneficia al sector transporte y el ahorro de horas-hombre

Esta obra es la realidad de un proyecto de inversión con código número 96799 en el Sistema Nacional de Inversión Pública (SNIP) del Ministerio de Economía y Finanzas (MEF). Esta obra va a facilitar el transporte de ayuda humanitaria y no humanitaria entre estos distritos en caso de un evento adverso. Surge la pregunta, ¿Qué proyectos de inversión de Gestión de Riesgo de Desastres existen en el Rímac?

En el 2018, es importante añadir la tendencia migratoria regional de países representada sustancialmente por la comunidad venezolana con un registro de más de 431 mil migrantes concentrándose principalmente en la ciudad de Lima y en sus distritos. Este hecho dificulta conocer la cantidad asentada en el Distrito del Rímac variando los índices de habitantes por km2, y otras cifras estadísticas para el cálculo de estimaciones de cualquier ayuda en caso de desastre. (05 de octubre del 2018. "Venezolanos en Perú: cifras actualizadas de la migración venezolana. El Comercio.).

\section{Gestión de Riesgos de Desastres en Lima}

La Gestión de Riesgo de Desastres en las zonas urbanas está relacionada con una serie de conceptos como planes de Desarrollo Urbano, Planes de Operaciones de Emergencia, Ciudades Sostenibles, Objetivos de Desarrollo Sostenible, Ordenamiento Territorial, Conurbación Urbana de las ciudades de Lima y-Callao, Proyectos de Inversión, y Obras por impuestos, 
entre otros. Su finalidad es la mejor calidad de vida y seguridad del sustento de la población.

Esta Gestión requiere un análisis de la interacción compleja del crecimiento poblacional, de la expansión de superficie habitable, de la demanda de provisión de alimentos, de la demanda de recursos para su industria del estrés hídrico, de la disminución de la zona de cultivos, y de la demandada zona de destino final de los desperdicios.

También se considera la normatividad legal, los tipos de proyectos de inversión de obras públicas incorporando un análisis de los riesgos de desastres, la transición de las autoridades públicas cuando terminan sus periodos de gobierno local, regional y/o municipal y los casos de corrupción en obras públicas.

No existen los desastres naturales. Los fenómenos naturales no causan un desastre. Son los impactos de los fenómenos naturales y las acciones humanas descontroladas en las zonas pobladas donde no ha existido, ni se ha desarrollado una cultura de prevención, de respeto a la convivencia con la naturaleza que genera un desastre en la población y/o en su fuente de vida de la población.

En 1970, con el fenómeno natural combinado de terremoto y aluvión en el callejón de Huaylas en Ancash que destruyó la ciudad de Huaraz, surge y se difunde el concepto de Defensa Civil con el Sistema Nacional de Defensa Civil (SINADECI). En el 2011, según norma publicada en El Peruano (Ley 29664. 2011. Art 5.3.h y 6) se crea el SINAGERD que diferencia tres componentes: la gestión prospectiva, la gestión correctiva y la gestión reactiva, e involucra a los tres niveles de gobierno (nacional regional y local). En el nivel local de las municipalidades se desarrolla esta labor en el área de Defensa Civil que cambia su denominación a Gestión de Riesgo de Desastres

Dentro del SINAGERD, se crean las siguientes entidades Centro Nacional de Estimación, Prevención y Reducción del Riesgo de Desastres (CENEPRED), Instituto Nacional de Defensa Civil (INDECI), y el Centro Nacional de Planeamiento Estratégico (CEPLAN).

En el reglamento de la ley de SINAGERD están definidos como entidades de respuesta (a) Fuerzas Armadas. (b) Policía Nacional (PNP). (c-) 
Cuerpo General de Bomberos Voluntarios del Perú. (d). Sector Salud (Ministerio de Salud, Seguro Social de Salud - ESSALUD, Instituciones Privadas de Salud, Sanidad de las Fuerzas Armadas (FFAA) y Sanidad de la PNP. (e.) Ministerio de la Mujer y Desarrollo Social. (f). Cruz Roja Peruana. (g.) Población organizada. (h). Otras entidades públicas y privadas que resulten necesarias. (El Peruano. Ley 29664. 2011. Art 46.2)

En el 2007, el MEF (2007. p. 8 de 100) ya había previsto la necesidad que, en las inversiones realizadas con recursos públicos, se incorpore el Análisis del Riesgo para contribuir a la sostenibilidad de estas inversiones.

En GRD, el MEF tiene asignado el programa presupuestal 0068 de Reducción de Vulnerabilidad y atención de emergencias por desastres para los tres niveles en el gobierno nacional, regional y gobiernos locales. (MEF 2018. Estructura programática.).

La GRD está ubicada en el organigrama del municipio del Rímac en la Sub-Gerencia de Gestión de Riesgo de Desastres que depende de la Gerencia de Desarrollo Económico y Turístico. En cambio, en la Municipalidad Metropolitana de Lima está ubicada en la Gerencia de Defensa Civil y Gestión del Riesgo de Desastres. Es decir, su ubicación organizacional a nivel gerencia o sub-gerencia implica diferentes niveles de decisión y acción y de montos de presupuestos públicos. (MDR. 2018. Organigrama.) (MML 2018. Organigrama.)

El Centro Nacional de Estimación, Prevención y Reducción del Riesgo de Desastres (CENEPRED), una de las entidades componentes del SINAGERD ha desarrollado un visor cartográfico del Perú que es una aplicación Sistema de Información Geográfica(SIG)que tiene capas de elementos expuestos (instituciones educativas, comisarias, almacenes), infraestructura vial y transporte, infraestructura eléctrica, infraestructura hídrica. Aparentemente ese visor de libre acceso es un producto final.

Sin embargo, este visor podría incluirse más data en sus capas, de las torres aéreas de transformación de voltaje en la infraestructura eléctrica, de los hidrantes en la infraestructura sanitaria, de los locales con cilindros de gas licuado de petróleo de 500 y 1.000 galones en la infraestructura para hidrocarburos. (CENEPRED 2018). Esta data proyecta las condiciones de peligro potenciales en la zona. 
Del 2007, se tiene la experiencia de las zonas de albergue luego del sismo de Pisco en el departamento de Ica. Los albergues eran los parques y calles. Así mismo, se reportaron alrededor de 100 albergues en la región de Ica, sumando un total de 6,014 familias albergadas, representando a 24,073 personas.

Según la Oficina de Coordinación de Asuntos Humanitarios de las Naciones UNIDAS (NNUU-OCHA) (2008. p. 17, 30 de 52). La experiencia en Pisco fue de múltiples dimensiones, por ejemplo: (1) Social por el hacinamiento, (2) Psicológico por la presencia de problemas de depresión, ansiedad, y (3) De seguridad ciudadana por problemas de delincuencia por la falta de protección perimetral. Este documento recomienda la capacitación del Proyecto Esfera para los administradores de los albergues).

En el 2009, en un evento internacional desarrollado por INDECI (2009. p. 176-177 de 234)de Lecciones del Sur por el Sismo de Pisco con la participación de 160 representantes de entidades internacionales y nacionales(del sector público y privado) se analizaron distintos componentes, por ejemplo, se llega al consenso de serias deficiencias, en la planificación, en la ubicación, en la gestión, en la participación de los actores en el liderazgo, en la coordinación, y en la implementación de los albergues.

Ante la magnitud del sismo de Pisco intervino el gobierno nacional en la etapa post-desastre porque el gobierno local municipal no tenía los recursos necesarios.

Hasta el 2018, no hay registros públicos de simulaciones, ni de simulacros de albergues.

\section{El Rímac, la GRD y un desastre}

El Rímac tiene cuatro focos de desarrollo comercial: en la zona de Jr. Trujillo, en Caquetá, en el cruce de Jr. Pizarro y prolongación Tacna y en la Av. Alcázar. En su infraestructura de salud; en el distrito hay seis centros de salud y un puesto de salud. En su infraestructura de seguridad hay cuatro comisarías y el cuartel militar Hoyos Rubio MDR. 2016. p 75,76 y 79 Plan de Desarrollo Concertado). 
Según un estudio del Instituto Geológico, Minero y Metalúrgico (INGEMMET) (2009, p. 18) en el Cerro San Cristóbal se tiene zonas habilitadas donde "se observa bloques en forma errática, subredondeados, ubicados en ladera, llegando a medir hasta 2 metrosde diámetro, estos se han originado por el fracturamiento amplio de la roca. Ladera con fuerte pendiente Estos bloques ante un sismo pueden ceder Material suelto en las laderas, generados por acción antrópica (construcción de vías de acceso y de viviendas”.

Desde el 2015, en el distrito del Rímac se registra una expansión urbanística con: (1) El Condominio Alcázar (2) El condominio Alcázar Besco en la zona donde funcionaba el Club Internacional Revolver. Los condominios tienen edificaciones de hasta 8 niveles. (3) El Condominio parque Rímac, referencia arque San Germán. (4) El proyecto Los Jardines del Rímac en Av. Jorge Bravo Rueda 266-280. Estas obras aumentan la densidad poblacional por sectores e incrementa la demanda de zonas de albergue $y$ refugio en casos de desastre.

El distrito del Rímac ha experimentado eventos que fuera de control y sin medidas de prevención han ocasionados desastres, por ejemplo, un gran incendio en Cantagallo en el 2016, el desborde del rio Rímac por el fenómeno del Niño en 2017 y el sismo de 7,9 grados con epicentro en la zona de Pisco-Ica del 2017 con impactos negativos en la zona con edificaciones antiguas del Rímac.(01 de febrero del 2017. Río Huaycoloro se desborda, inunda SJL y llega al Rímac. La República.).

El 04.11.2016 hubo un incendio en la zona denominada Cantagallo habitada por la comunidad nativa de la selva de la etnia Shipibo-Coniba asentada en el distrito del Rímac. Según cifras aproximadas fueron damnificadas 389 familias, conformadas por 1721 habitantes y con el fallecimiento de un menor de 11 años por quemaduras de tercer grado.

Esta emergencia generada por un incendio estaba localizada en un solo distrito. Por tanto, solo era necesario la activación del Centro de Operaciones de Emergencia (COE) del Rímac que actúa a nivel municipal. Los niveles de emergencia se califican según el impacto y la capacidad de respuesta Véase tabla 3.

En la gestión reactiva estuvieron presente las autoridades municipales del Rímac y de Lima Metropolitana a través de sus respectivos COEs. 
Desde 1990, según un estudio de la Defensoría del Pueblo (2016)cita que la comunidad indígena Shipibo-Coniba estaba asentada en la zona de Cantagallo. En junio del 2007, el municipio del Rímac los reconoce en calidad de posesionarios del terreno donde habitaban en el Rímac. En el 2009, MML gestiona una obra vial denominada Línea amarilla (luego Vía Parque Rímac) que impactaría a la comunidad Shipibo-Coniba. Esta circunstancia explica la activación del COE-MML.

Tabla 3

Niveles de Emergencia

\begin{tabular}{|c|c|c|c|c|c|}
\hline CRITERIOS & NIVEL 1 & NIVEL2 & NIVEL 3 & NIVEL 4 & NIVEL 5 \\
\hline $\begin{array}{l}\text { Cobertura } \\
\text { geográfica }\end{array}$ & $\begin{array}{l}\text { Emergencia } \\
\text { localizada en un } \\
\text { solo Distrito. }\end{array}$ & $\begin{array}{l}\text { Emergencia } \\
\text { localizada en dos } \\
\text { o más Distritos. }\end{array}$ & $\begin{array}{l}\text { Emergencia } \\
\text { localizada en dos } \\
\text { o más Provincias. }\end{array}$ & $\begin{array}{l}\text { Emergencia } \\
\text { localizada en dos } \\
\text { o más Regiones. }\end{array}$ & $\begin{array}{l}\text { Emergencia } \\
\text { localizada en dos o } \\
\text { más Regiones. }\end{array}$ \\
\hline Impacto & Local/Distrital & $\begin{array}{l}\text { Distrital/ } \\
\text { Provincial }\end{array}$ & $\begin{array}{l}\text { Regional/ } \\
\text { Metropolitano }\end{array}$ & Macro-Regional & Nacional \\
\hline $\begin{array}{l}\text { Capacidad de } \\
\text { respuesta }\end{array}$ & Distrital & Provincial & $\begin{array}{l}\text { Regional/ } \\
\text { Metropolitano }\end{array}$ & Nacional & Internacional \\
\hline $\begin{array}{l}\text { Centro de } \\
\text { Operaciones de } \\
\text { Emergencia }\end{array}$ & COED & COEP & COER & COEN & COEN-OSOCC \\
\hline $\begin{array}{l}\text { Tiempo referencial } \\
\text { de atención que } \\
\text { demanda la } \\
\text { emergencia }\end{array}$ & $\begin{array}{l}\text { Tiempo máximo } \\
\text { de } 30 \text { días }\end{array}$ & $\begin{array}{l}\text { Tiempo máximo } \\
\text { de } 90 \text { días }\end{array}$ & $\begin{array}{l}\text { Tiempo máximo } \\
\text { de } 150 \text { días. }\end{array}$ & $\begin{array}{l}\text { Tiempo mínimo } \\
\text { de } 180 \text { días } \\
\text { (aproximada- } \\
\text { mente medio año }\end{array}$ & $\begin{array}{l}\text { Tiempo mínimo } \\
360 \text { días (aproxi- } \\
\text { madamente } 1 \text { año } \\
\text { a más }\end{array}$ \\
\hline
\end{tabular}

OSOCC $=$ On-Site Operations Coordination Centre

Fuente: El Peruano. 2011. Decreto Supremo 048-2011-PCM artículo 43, INDECI -MML-POE 2015. p. 45 de 281

La población damnificada y afectada por este evento adverso fue reubicada en el campo deportivo denominado Martinete en el sector de Barrios Altos en el Cercado de Lima. Es decir, es un espacio físico fuera del distrito del Rímac quedando en evidencia que la municipalidad del Rímac no tenía un espacio disponible ni asignado para atender a esta comunidad de 1.721 habitantes luego de un desastre.

Desde el 2016, el Municipio del Rímac (2016, p 19-22 de44) tiene un Plan de Rehabilitación ante Riesgos de Desastres para el distrito del Rímac. Este documento tiene una relación de 33 zonas seguras y 36 puntos de concentración y ninguno de estos fue usado para atender este siniestro del incendio. Este mismo documento no explica porque fueron identificados 
ni seleccionados estos lugares ni que cumplan algún parámetro técnico ni que fueran validados por alguna entidad técnica además de ser espacios libres y/o áreas verdes. No aparece información del COE-Rímac sobre estos lugares en sus reportes del incendio de Cantagallo.

Surgen las siguientes interrogantes,

- ¿Pueden los parques de un distrito ser usados como zonas de refugio?

- ¿Los usos de suelos o los planos de zonificación son decididos libremente por los municipios?

- ¿Porque en el plano de zonificación elaborado por el Instituto Metropolitano de Planificación (IMP) y aprobado para el distrito del Rímac no existe un concepto afín al Riesgo de Desastre?

- ¿Existe en algún distrito?

- ¿Es una buena respuesta de atención al desastre el uso forzado de parques, campos deportivos, colegios, y templos entre otros?

- ¿Conocen las autoridades locales y la sociedad civil para qué sirve el Proyecto Esfera?

- ¿A cuántas personas pueden albergar estas zonas de albergue?

Respecto de los lugares identificados por la MDR como zonas seguras y puntos de concentración se tiene por ejemplo a la Alameda de los Bobos donde existe una estación de combustible y un transformador aéreo de medio voltaje que representan un riesgo de explosión de combustible y un riesgo de explosión de descarga eléctrica respectivamente. Y el hecho de ubicar a personas afectadas aumenta la probabilidad que sean más las personas afectadas. Entonces se concluye que esta supuesta zona segura es realmente una zona insegura.

Surge la pregunta, ¿Estos lugares que fueron seleccionados por su autoridad local, fueron validados técnicamente por las otras entidades competentes en el tema de GRD, por ejemplo, INDECI, CENEPRED, Colegio de Ingenieros del Perú? ¿Es su funcionario responsable del área de GRD municipal una persona con un perfil profesional que corresponda al cargo? 
Por ejemplo, en el punto de concentración establecido en Jr. Trujillo, las edificaciones de hasta tres niveles son de material de quincha y adobe, y por su antigüedad y falta de mantenimiento representan un peligro por el riesgo de derrumbe.

En el caso de una emergencia de nivel $N^{\circ} 2$ o superior, ¿Cómo un equipo humano de respuesta de otro distrito, que tenga que movilizarse hacia el distrito del Rímac y desconozca sus direcciones, puede encontrar una "zona segura" en la dirección de Calle SN en el sector Laderas del Barrio Los Ángeles y de nombre SN donde SN significa sin nombre?

Este incendio afectó a una comunidad de aproximadamente 2.000 habitantes y hubo dificultad en su albergue. Surge la interrogante, ¿Qué sucederá con la capacidad de albergues, puntos de concentración, zonas seguras cuando un evento adverso genere una mayor cantidad de habitantes afectados y damnificados, por ejemplo, con los pobladores de las laderas del cerro San Cristóbal, o con los asistentes concentrados en el Coliseo de Acho con capacidad de 13.000 personas ante un sismo de magnitud 8,0 ?

En el distrito del Rímac, los lugares con potencial de albergar mayor cantidad de ciudadanos son el Coliseo de Acho y el parque zonal Cápac Yupanqui. Este parque zonal tiene un área de 31.749,39 $\mathrm{m}^{2}$ y está bajo la administración de Servicio de Parques de Lima (SERPAR) de la MML.

Entonces será necesaria la ayuda externa de otros niveles del COE. Como podrá llegar la ayuda humanitaria y no humanitaria a los puntos de concentración y las zonas seguras si son difíciles de ubicar e incluso con restricciones de tránsito para vehículos de carga pesada como contenedores.

En la etapa de Gestión Reactiva, la población afectada con una calidad de vida seriamente disminuida puede ocasionar un conflicto social por el tiempo prolongado en recuperar sus formas de vida. También, se debería establecer plazos del inicio de la etapa de Reconstrucción socioeconómica.

El problema se agrava si se revisa los informes del COE-Rímac donde no hay datos de la aplicación del Proyecto Esfera durante el tiempo de ayuda a las personas afectadas. Esta recomendación respecto del Pro- 
yecto Esfera surgió dentro de las lecciones del sur en el evento de INDECI (2009. p. 176-177 de 234) y se puede inferir que no ha sido establecida como un procedimiento dentro de los COE ni en los talleres de capacitación del área de GRD municipal.

Según el documento de INDECI (2011. p. 325-329), el Plan de Operaciones de Emergencia (POE) para Lima y Callao del 2011 plantea en la fase ayuda humanitaria un procedimiento de empadronamiento, instalación y manejo de albergues, y de reunificación familiar. También se incluiría el requerimiento de personal, vehículos, y un área adicional para ser utilizada por estos recursos. Además estos recursos no deberían ocupar las vías de tránsito.

La administración municipal del Rímac en el área de GRD muestra en su página web el desarrollo de un visor cartográfico con aplicaciones de tipo SIG denominado "proyecto RIMAC-DRR "Reinforcing Innovative Mechanisms for Arising Capacities in Disaster Risk Reduction in Rímac" pero al ingresar al enlace respectivo aparece un mensaje de error. Es decir, un servicio que no está funcionando.

Surgen nuevas interrogantes: ¿Porque se desarrolla un visor cartográfico en la MDR cuando debería aprovecharse el visor cartográfico existente desarrollado por CENEPRED? ¿Porque no se verifica la coordinación interinstitucional en el sector estatal en este recurso informativo?

\section{Respuesta parques vs albergues}

El caso expuesto del evento adverso del incendio fuera de control que afectó la comunidad Shipibo-Coniba en un lugar físicamente delimitado demuestra varios aspectos - la falta de espacios adecuados para atender a la población afectada, la falta de difusión pública de correcciones en las coordinaciones interinstitucionales, la presencia de elemento político en una actividad técnico-operativo de ayuda humanitaria y no-humanitaria a la población afectada y damnificada, entre otros.

Según la magnitud del evento adverso, en estas zonas de albergue, de refugio debe planificarse un área de atención médica en caso de que la capacidad de la infraestructura hospitalaria en el distrito sea insuficiente. 
Los eventos adversos se registran en el Sistema de Información Nacional para la Respuesta y Rehabilitación (SINPAD) con alcance a entidades Públicas o Privadas, así como por la ciudadanía en general, según su página. Sin embargo, el ciudadano común no tiene acceso a este sistema, incluso ni acceso al servicio de internet. (SINPAD. 2018. Aplicaciones).

Entonces en la página web de la MDR no se encuentra una prueba de una coordinación pública ni uso del visor Sistema de Información para la Gestión de Riesgo de Desastres (SIGRID) de CENEPRED, ni del SINPADINDECI. No se encuentra una sección de enlaces con otras entidades como prueba de una coordinación interinstitucional.

La comunidad indígena Shipibo-Coniba reside en la ciudad de Lima, desde hace 28 años. Equivale a que tienen una generación que ha nacido, crecido, educado con las costumbres pluriculturales en la ciudad de Lima, donde hay un reemplazo de la naturaleza por el cemento, por eso su reasentamiento poblacional definitivo tiene complejidad porque es un tema cultural, étnico, socio-económico y político. Y como tema político tiene un desarrollo a largo plazo.

El tema del artículo se centra en resaltar la importancia acerca de que cualquier grupo humano requiere ser atendido, albergado en forma temporal después de un desastre. En el caso presentado fue esta comunidad Shipibo-Coniba que al margen de sus antecedentes de un proceso de reasentamiento definitivo tiene una necesidad inmediata de zonas de refugios/albergues en su condición de afectados y/o damnificados por un evento adverso.

Una parte de la problemática es reubicar a esta población afectada/ damnificada que es una de las variables a identificar que debería analizarse para corregir la gestión del estado y operatividad de los refugios/ albergues de la zona distrital.

En 1997, el proyecto Esfera aparece como una guía internacional para ayudar a las poblaciones afectadas y damnificadas por desastres a recuperar sus condiciones de calidad de vida. Dentro de estas guías aparece el componente de las zonas de albergue temporal. - En Chile y Filipinas, este proyecto Esfera ha sido integrado dentro de sus sistemas de respuesta a través del Programa de las Naciones Unidas para el Desarrollo (PNUD) 
empleando indicadores para las normas mínimas en refugio, y seguridad alimentaria y nutrición. (26 de octubre del 2017. Localización de Esfera: Chile y Filipinas integran las normas Esfera en sistemas nacionales de respuesta a emergencias. Proyecto Esfera.)(Proyecto Esfera. 2011. p. 29 a 52 de 450).

El Proyecto Esfera (1999 p. 2 y 9 de 450) o la Carta Humanitaria y normas mínimas para la respuesta humanitaria fue elaborado por organismo no gubernamentales y el movimiento Internacional de la Cruz Roja, entre otros.

1. Tiene "el objetivo de mejorar la calidad de las respuestas humanitarias en situaciones de desastre o de conflicto y mejorar la rendición de cuentas del sistema humanitario ante la población afectada por el desastre". Es asegurar Vida con dignidad, y aliviar el sufrimiento traumático en el post-desastre.

2. Presenta dos partes (1) Los principios que comprende la carta humanitaria, los principios de protección, y las normas esenciales, y (2). Las normas mínimas que abarcan el abastecimiento de agua, el saneamiento y promoción de la higiene, la seguridad alimentaria y nutrición, el alojamiento asentamientos humanos y artículos no alimentarios y acción de salud.

La importancia del conocimiento, aprendizaje y aplicación del Proyecto Esfera en estos lugares de albergue y refugio tiene un componente emocional de alivio por el cambio brusco de forma de vida de la población. Un albergue mal administrado con la ayuda humanitaria y no-humanitaria va a generar conflictos socio-económicos y va agravar las condiciones de vida en estas zonas de albergue.

En el 2018, no hay registros públicos en la web de la MDR del conocimiento y capacitación del Proyecto Esfera dentro de su Plan de Ayuda en caso de desastre, ni en su informe distrital del COE en el caso del incendio de Cantagallo. (24 de noviembre del 2016. "Las acciones ejecutadas y Situación actual de Cantagallo tras incendio del 04 de noviembre”. MDR.).

En el 2018, no hay registros de un seguimiento a los estados operativos de sus parques y áreas verdes en la MDR. Existen casos en otros distritos de uso inadecuados de estas zonas verdes, por ejemplo una supuesta construcción ilegal en un parque de San Juan de Miraflores y el in- 
terés de construir un centro comercial en Comas. (01 de agosto del 2018). "Vecinos protestan contra supuesta construcción ilegal en parque de SJM". Peru21.) (09 de noviembre del 2016. "Comas quiere convertir área de parque en centro comercial”. El Comercio.).

\section{Reflexión, cambios urgentes}

¿Cuál sería el impacto por los fenómenos naturales que causan desastres de magnitud severa en una zona urbana que no tiene zonas de albergue ni lugares que pueden ser usados como zonas de albergue?

Las personas damnificadas y afectadas estarían dispersas, las familias no tendrán un lugar seguro para reunificación, se complicaría la logística para atenciones de salud, para identificaciones de fallecidos, para la recepción y distribución de la ayuda humanitaria y no humanitaria, ni lugar donde protegerse del clima con lluvias, frio o sol intenso, entre otros casos. Además, harían uso forzado como zonas de albergues de las escuelas.

Existe una aparente fragmentación en la cadena de información, si bien el MDR tiene un plan de reconstrucción, y tiene zonas de albergue designadas; no hay información que estas zonas tengan condiciones técnicas ni que los responsables de GRD conozcan de su operatividad.

\section{El factor normativo-técnico.}

La operatividad, efectividad y el buen funcionamiento de las zonas de albergue va a depender de varios factores:

- De la disponibilidad de terreno, que cumpla condiciones técnicas estándar, accesibilidad de las zonas de albergue.

- De los requerimientos de personal que cumpla con un perfil profesional para la operatividad de estas zonas de albergue.

- De los funcionarios públicos que deben conocer y hacer funcionar además de estas zonas, a todos los demás componentes del sistema GRD, de la capacitación, de las alertas, de los simulacros de eventos, de las coordinaciones con las otras entidades de respuesta. 
- De la información de los componentes de la GRD en el distrito.

- De casos de corrupción de tráfico ilícitos de terrenos y de parques invadidos en otros distritos que representan un potencial peligro.

- Que la población local los localice e identifique.

- Que las entidades y autoridades no locales puedan ubicarlos cuando requieran buscarlos para el arribo de la ayuda humanitaria y no humanitaria.

- De ejecutar simulaciones del funcionamientos de estas zonas para detectar y corregir fallas de operación y de coordinación entre población y funcionarios locales y actores externos.

Es importante que el personal que atienda estos lugares durante el post-desastre sea entrenado en el apoyo emocional que requieren las personas y familias separadas, afectadas y damnificadas por el estrés generado por el desastre.

Desde el 2016, la MDR tiene un plan de rehabilitación ante riesgo de desastres. En el 2016, se produjo el incendio de Cantagallo. En el 2018, no hay información pública de evaluación de la eficiencia de ese plan en contraste con ese evento adverso. Es decir, si funcionó o no funcionó, ya sí saber que componentes requieren fortalecimiento.

Los cambios de las principales autoridades municipales, los Alcaldes, se produce en ciclos de cuatro (4) años. Los últimos periodos han sido: 2011-2014, 2015-2018, 2019-2022.De tal forma que desde la creación de SINAGERD hasta la elaboración del plan municipal en el Rímac fue durante la gestión de dos alcaldes, dos periodos municipales. Y el personal de la subgerencia de Defensa Civil es un cargo de confianza, hecho que no implica que sea personal calificado en el campo de Gestión de Riesgo de Desastre.

De los responsables/funcionarios públicos, del área de GRD en la MDR se apreció una alta rotación laboral en este periodo de gobierno municipal del 2015-2018, Este factor de cambio implica reiniciar todo el sistema administrativo con cada nuevo jefe. Además, no existe un perfil profesional 
definido y estandarizado para este puesto. ¿Y siendo un cargo de confianza, puede desempeñarlo una persona sin conocimiento en el tema de GRD?

Sería útil, que los responsables de la GRD del MDR analicen la ubicación y operatividad de nuevas zonas de albergue considerando que el crecimiento urbano poblacional es dinámico. Y que en coordinación con otras entidades estatales como la Superintendencia Nacional de Bienes Estatales (SBN), la Superintendencia Nacional de los Registros Públicos (SUNARP), y el Ministerio Publico identifiquen terrenos disponibles, o invasiones ilegales de áreas públicas o privadas.

\section{Voluntariado}

Otro dato relevante, es la interacción de las autoridades públicas locales con la sociedad civil. En el marco normativo nacional y local, se establece un voluntariado. Sin embargo, de esta actividad no tiene registro ni en noticias periodísticas ni en el informe del COE distrital

Esta interacción de ejecutar la ayuda en la zona de albergues depende de responsables en cada entidad. Por tanto, se requiere talleres para plantear la importancia de la participación del voluntariado civil, y luego evaluar su participación dentro de los simulacros de sismos dentro del distrito.

Dentro de estos grupos de voluntariado, están los clubes departamentales, la población universitaria, las congregaciones religiosas, los scouts, juntas vecinales, agrupaciones comerciales, entre otros.

Surge la interrogante, ¿Cuál es su nivel de comunicación, coordinación y articulación de las entidades de Primera Respuesta a nivel municipal en GRD?

Sería útil desarrollar y reforzar este componente de los grupos de voluntariado y capacitarlos dentro del Proyecto Esfera.

\section{El factor financiero}

Dentro del presupuesto para la GRD, la inversión en albergues/zonas de refugio es imprecisa. El programa presupuestal 0068 del MEF de Reducción de la vulnerabilidad y atención de emergencias por desastres 
tiene un concepto de instalación denominado TRANSPORTE E INSTALACION DE INFRAESTRUCTURA MOVIL, PUENTES MODULARES Y ALBERGUE. Este concepto de instalación asume que existe el lugar físico para instalar la zona de albergue. Es un gran supuesto que falla dentro de la planificación. (MEF. 2018. Estructura programática.) (MEF. 2018. p. 1 a 11).

En el 2016, la experiencia del municipio de San Isidro gestionó como elemento complementario en la cadena de abastecimiento a las zonas de albergue, y de refugio un proyecto de inversión de la construcción de un almacén subterráneo para abastecer a los albergados. La MDR podría ejecutar una experiencia similar.

En resumen, de la operatividad del SINAGERD, se tiene el Recurso financiero del Programa Presupuestal PP $N^{\circ} 0068$ pero no se tiene el detalle desagregado del monto destinado a zonas de albergues, se tiene el recurso legal de la creación del SINAGERD y el área municipal de GRD pero el recurso humano asignado tiene una alta rotación laboral y no se asegura que cumpla un perfil profesional técnico, se tiene el recurso material de los parques pero estos son lugares forzados para su uso como albergues. Se tiene el recurso administrativo de planificación pero no es evaluado periódicamente ni hay datos de su concertación con otras entidades.

En el 2021, Perú cumple su bicentenario como república, el distrito del Rímac cumple su primer centenario, y el SINAGERD cumple 10 años. El Perú y el Rímac tienen problemáticas complejas a nivel nacional y a nivel local respectivamente. En el caso de SINAGERD, que es transversal a los sectores económico, vivienda entre otros se requiere más tiempo que un periodo de gobierno local/municipal para una mayor evolución que asegure mejor operatividad en todos sus componentes.

El Rímac no puede expandirse requiere redefinir su espacio urbanístico incluyendo en sus nuevos proyectos de inversión el componente de Gestión de Riesgo de Desastres y ejerciendo su autonomía política, económica y administrativa municipal en un trabajo coordinado y articulado de un proceso de Ordenamiento Territorial a cargo del MINAM (2014 p. 9), y también del MEF, de MML, y de instituciones técnicas como CENEPRED, INDECI e INGEMMET. 


\section{Glosario de términos}

Definiciones extraídas. INDECI. 2005. p 509 a 512 de 512.

Afectado.- Persona, animal, territorio o infraestructura que sufre perturbación en su ambiente por efectos de un fenómeno. Puede requerir de apoyo inmediato para eliminar o reducir las causas de la perturbación para la continuación de la actividad normal.

Damnificado.- Persona afectada parcial o íntegramente por una emergencia o desastre y que ha sufrido daño o perjuicio a su salud o en sus bienes, en cuyo caso generalmente ha quedado sin alojamiento o vivienda en forma total o parcial, permanente o temporalmente, por lo que recibe refugio y ayuda humanitaria temporales. No tiene capacidad propia para recuperar el estado de sus bienes y patrimonio.

Definiciones extraídas. MDR (2016. p 19-22 de 44).

Puntos de Concentración: Son lugares de abastecimiento temporal de agua potable y alimentos; así como, la atención y primeros auxilios definidos por la autoridad local de Defensa Civil y a donde deberás dirigirte en caso de un sismo de gran magnitud.

Zonas de Seguridad Externa: Son los parques, plazas, áreas verdes o campos deportivos u otra área libre determinada por la Subgerencia de Gestión de Riesgo de Desastre y Defensa Civil.

\section{Referencias Bibliografías}

Congreso de la República del Perú. Publicada en El Peruano (2011). Lima-Perú. Ley № 29664 del 19 de febrero del 2011. Ley que crea el Sistema Nacional de Gestión del Riesgo de Desastres (SINAGERD). Artículos Art 5.3.h y 6).

Congreso de la República del Perú. Publicada en El Peruano (2011). Lima-Perú. Decreto Supremo No 048-2011-PCM del 26 de mayo del 2011. Reglamento de la Ley № 29664, Ley que crea el Sistema Nacional de Gestión del Riesgo de Desastres (SINAGERD). Artículo 46‥- Primera Respuesta.

INDECI (2005). Compendio Estadístico de Prevención y Atención de Desastres. Lima-Perú Edición 1ra. p.509 a 512 de 512. 
IMP (2010). Inventario de Áreas Verdes a nivel metropolitano, Lima-Perú. p. 6-7 de 46.

MEF (2007). Pautas metodológicas para la incorporación del análisis del riesgo de desastres en los Proyectos de Inversión Pública. Lima-Perú. Edición 1ra. p. 8 de 100.

MINAM (2014). Orientaciones básicas sobre el Ordenamiento Territorial en el Perú. Lima-Perú. p 19 de 60.

NNUU-OCHA (2008). Diagnóstico de necesidades no cubiertas en la población afectada por el terremoto del 15 de agosto del 2007en el Departamento Ica, Perú. Ica-Perú Edición 1ra. p. 17 de 52.

INDECI (2009). Lecciones Aprendidas del Sur - Sismo de Pisco, 15 agosto 2007. Lima-Perú. Edición 1ra. p. 176-177 de 234.

INGEMMET (2009). Zonas Críticas por peligros geológicos en Lima Metropolitana Cuadro de zonas críticas ítem 30. Lima-Perú. p. 18 de 38.

INDECI, PNUD, otros. (2011). Plan de Operaciones de Emergencia para el área

Metropolitana de Lima y la Región Callao. Lima-Perú. Edición 1ra. p. 325-329 de 329.

Proyecto Esfera (2011). Carta Humanitaria y normas mínimas para la respuesta humanitaria. Northampton, United Kingdom, Edición 3ra p. 29 a 52 de 450.

MDR (2016). Plan de Rehabilitación ante Riesgo De Desastres En el Distrito del Rímac. Lima-Perú, p. 19-22 de 44

MDR (2016). Plan de Desarrollo Concertado. Lima-Perú.p 75, 76 y 79 de306

MEF (2018). Proceso Presupuestario del Sector Publico- Programa Presupuestal Producto / Proyecto Actividad / Obra-Combinación de Cadenas Funcionales Programas Presupuestales 2018. Lima-Perú. p. 1 a 11

UNICEF (2008). Escuelas como albergues. ¿Cuándo?, ¿Cómo?, ¿Porque? p 1-68 